\title{
New examples of curves with a one-dimensional family of pencils of minimal degree
}

\author{
Nils HenRy Williams Rasmussen
}

\begin{abstract}
We give a geometric construction of sub-linear systems on a K3 surface consisting of smooth curves $C$ with infinitely many $g_{\operatorname{gon}(C)}^{1}$ 's.

Mathematics Subject Classification (2010). Primary 14H51;

Secondary $14 \mathrm{~J} 28$.
\end{abstract}

Keywords. Curves on K3 surfaces, gonality, Brill-Noether theory.

1. Introduction. Let $C$ be a smooth curve over $\mathbb{C}$ of genus $g \geq 2$. One defines $W_{d}^{r}(C)$ to be the variety parametrising complete $g_{d}^{r^{\prime}}$ 's on $C$ such that $r^{\prime} \geq r$. The expected dimension is $\rho(g, r, d):=g-(r+1)(g-d+r)$, and is the dimension of $W_{d}^{r}(C)$ when $C$ is general in $\mathscr{M}_{g}$, provided that $\rho(g, r, d) \geq 0$. We will be interested in finding new examples of smooth curves $C$ with an infinite number of pencils of minimal degree on certain K3 surfaces.

A number of results have been proved for curves on K3 surfaces throughout the last 25 years. In 1986, it was proved by Lazarsfeld [10] that if the linear system $|L|$ on a K3 surface $S$ doesn't contain non-reduced or reducible curves, then $\operatorname{dim} W_{d}^{r}(C)=\rho(g, r, d)$ for general $C \in|L|$. Knutsen [9] proved that the only cases of exceptional curves (i.e., curves $C$ satisfying $\operatorname{Cliff}(C)<\operatorname{gon}(C)-2)$ on K3 surfaces are the Donagi-Morrison example [3, (2.2)] and the generalised ELMS example (a generalisation of [4, Theorem 4.3] presented in Knutsen's article, see "Generalised ELMS examples"). He furthermore proved that the gonality is constant for all curves in a linear system on any K3 surface as long as it is not as in the Donagi-Morrison example. A weaker result of Knutsen's gonality theorem was proved in [1], for the case of ample linear systems on K3 surfaces.

Among the most recent results on curves on K3 surfaces, are one by Knutsen in $[8$, Theorem $1.1(\mathrm{~b})]$, proving that there exist curves $C$ of any possible gonality $\operatorname{gon}(C) \leq\lfloor(g+3) / 2\rfloor$ on K3 surfaces; and one in [9, Theorem 3.1], 
proving that whenever $|L|$ is a base-point free linear system on a K3 surface not as in the Donagi-Morrison example and the smooth $C \in|L|$ satisfy $\rho(g, 1, \operatorname{gon}(C))<0$, then $\operatorname{dim} W_{\operatorname{gon}(C)}^{1}(C)=0$ for the general smooth $C \in|L|$.

For the curves $C$ in the Donagi-Morrison and generalised ELMS examples, we have $\operatorname{dim} W_{\operatorname{gon}(C)}^{1}(C)=1$ in both cases, as is the case in general for all exceptional curves $C$, by [2, Corollary 2.3.1], and also all curves of odd genus and maximal gonality. All finite covers $C^{\prime}$ of curves $C$ of degree $z$ with $\operatorname{dim} W_{\operatorname{gon}(C)}^{1}(C)=1$ and $g\left(C^{\prime}\right) \geq \operatorname{gon}\left(C^{\prime}\right) \cdot(z-1)+z \cdot g(C)$ will also give us $\operatorname{dim} W_{\text {gon }\left(C^{\prime}\right)}^{1}\left(C^{\prime}\right)=1$, by the Castelnuovo-Severi inequality [6]. By [4, Remark 3.8], there are no other known examples of curves with 1 dimension of $g_{\operatorname{gon}(C)}^{1}$ 's. Moreover, as far as we know, the curves in the generalised ELMS example and the plane and bi-elliptic curves in the Donagi-Morrison example are the only cases known so far of curves on K3 surfaces with $\operatorname{dim} W_{\operatorname{gon}(C)}^{1}(C)=$ 1.

In this paper, we give the following further examples of smooth curves on K3 surfaces with 1 dimension of $g_{\operatorname{gon}(C)}^{1}$ 's. The curves given here are nonexceptional.

Theorem 1.1. Let $S$ be a smooth projective $K 3$ surface with $\operatorname{Pic}(S) \cong \mathbb{Z} H$, where $H$ is a smooth curve on $S$ with $H^{2} \geq 4$. Then for any integer $m \geq 3,|m H|$ contains a sub-linear system where the smooth curves $C$ satisfy $\operatorname{dim} W_{\operatorname{gon}(C)}^{1}(C)=1$.

2. The result. Let $C$ be a smooth curve over $\mathbb{C}$. One defines the gonality of $C$, denoted $\operatorname{gon}(C)$, to be the smallest $k$ such that there exists a $g_{k}^{1}$ on $C$. For $g(C) \geq 4$, the Clifford index of $C$ is defined to be

$\operatorname{Cliff}(C):=\min \left\{\operatorname{deg}(A)-2\left(h^{0}(C, A)-1\right) \mid h^{0}(C, A) \geq 2\right.$ and $\left.h^{1}(C, A) \geq 2\right\}$.

Hyperelliptic curves $C$ of genus $g=2$ are defined to have $\operatorname{Cliff}(C)=0$, and trigonal curves $C^{\prime}$ of genus $g=3$ are defined to have Cliff $\left(C^{\prime}\right)=1$.

By [2, Theorem 2.3], we have $\operatorname{Cliff}(C) \in\{\operatorname{gon}(C)-2$, gon $(C)-3\}$. Curves $C$ satisfying $\operatorname{Cliff}(C)=\operatorname{gon}(C)-3$ are called exceptional.

A $K 3$ surface is defined to be a smooth, projective surface $S$ over $\mathbb{C}$ satisfying $h^{1}\left(S, \mathscr{O}_{S}\right)=0$ and $\mathscr{O}_{S}\left(K_{S}\right) \cong \mathscr{O}_{S}$. By the adjunction formula, it follows that $L^{2}=2 p_{a}-2$, where $p_{a}$ is the arithmetic genus of the curves in $|L|$. By [9, Theorem 2], the only exceptional curves on K3 surfaces are the ones found in $[3,(2.2)]$ and $[9$, "Generalized ELMS Examples"] (the latter a generalisation of [4]). By [5, Theorem], the Clifford index of the smooth curves in a linear system on a K3 surface is constant.

Lemma 2.1. Suppose $C \in|L|$ is a smooth curve of gonality $k$ such that $C \sim$ $D_{1}+D_{2}$, where $D_{1}, D_{2}$ are two divisors on $S$ satisfying $h^{0}\left(S, \mathscr{O}_{S}\left(D_{i}\right)\right) \geq 2$ for $i=1,2$. Then $\operatorname{Cliff}(C) \leq D_{1} \cdot D_{2}-2$. It follows that if $C$ is non-exceptional, then $D_{1} \cdot D_{2} \geq k$.

Proof. We show that $\left.D_{1}\right|_{C}$ contributes to the Clifford index of $C$. Consider the exact sequence $0 \rightarrow \mathscr{O}_{S}(-C) \rightarrow \mathscr{O}_{S} \rightarrow \mathscr{O}_{C} \rightarrow 0$ tensored with $\mathscr{O}_{S}\left(D_{1}\right)$ :

$$
0 \rightarrow \mathscr{O}_{S}\left(-D_{2}\right) \rightarrow \mathscr{O}_{S}\left(D_{1}\right) \rightarrow \mathscr{O}_{C}\left(\left.D_{1}\right|_{C}\right) \rightarrow 0
$$


Taking global sections, we see that $h^{0}\left(C, \mathscr{O}_{C}\left(\left.D_{1}\right|_{C}\right)\right) \geq h^{0}\left(S, \mathscr{O}_{S}\left(D_{1}\right)\right)$, which is $\geq 2$. Tensoring with $\mathscr{O}_{S}\left(D_{2}\right)$ instead of $\mathscr{O}_{S}\left(D_{1}\right)$ gives us $h^{0}\left(C, \mathscr{O}_{C}\left(\left.D_{2}\right|_{C}\right)\right) \geq$ $h^{0}\left(S, \mathscr{O}_{S}\left(D_{2}\right)\right)$, which is also $\geq 2$. Since $\left.K_{C} \sim\left(D_{1}+D_{2}\right)\right|_{C}$, then $K_{C}-\left.D_{1}\right|_{C} \sim$ $\left.D_{2}\right|_{C}$, and so we conclude that $h^{1}\left(C, \mathscr{O}_{C}\left(\left.D_{1}\right|_{C}\right)\right) \geq 2$, so that $\left.D_{1}\right|_{C}$ contributes to $\operatorname{Cliff}(C)$. It follows that

$$
\begin{aligned}
\operatorname{Cliff}(C) & \leq \operatorname{Cliff}\left(\left.D_{1}\right|_{C}\right)=\operatorname{deg}\left(\left.D_{1}\right|_{C}\right)-2\left(h^{0}\left(C, \mathscr{O}_{C}\left(\left.D_{1}\right|_{C}\right)\right)-1\right) \\
& \leq \operatorname{deg}\left(\left.D_{1}\right|_{C}\right)-2\left(h^{0}\left(S, \mathscr{O}_{S}\left(D_{1}\right)\right)-1\right) \leq D_{1} \cdot C-2 \cdot\left(2+\frac{1}{2} D_{1}^{2}-1\right) \\
& =D_{1} \cdot D_{2}-2
\end{aligned}
$$

as desired.

Proof of Theorem 1.1. Let $S^{\prime}$ be a smooth projective K3 surface satisfying $\operatorname{Pic}\left(S^{\prime}\right) \cong \mathbb{Z} H^{\prime}$, where $H^{\prime}$ is a smooth curve on $S^{\prime}$ with $\left(H^{\prime}\right)^{2}=2 g-2 \geq 4$. Then by a classical result in [12], $H^{\prime}$ is very ample (this result is stated more clearly in [7, Theorem 1.1] with $k=1$, where it says in particular that if $H^{\prime}$ is not very ample, then there exists an effective divisor $D$ on $S^{\prime}$ satisfying $\left.D^{2} \leq 0\right)$. It follows that $\mathscr{O}_{S^{\prime}}\left(H^{\prime}\right)$ defines an embedding from $S^{\prime}$ into $\mathbb{P}^{g}$, which restricted to any smooth curve $C^{\prime} \in\left|H^{\prime}\right|$ is the canonical embedding of $C^{\prime}$ into a hyperplane of $\mathbb{P}^{g}$. Note that the smooth curves in $\left|H^{\prime}\right|$ are non-hyperelliptic. Let $S$ be the image of $S^{\prime}$, and let $H$ be the image of $H^{\prime}$.

Now consider a smooth plane curve $X$ of degree $m \geq 3$ lying inside a plane $F \subseteq \mathbb{P}^{g}$, let $T \subseteq \mathbb{P}^{g}$ be a codimension 3 linear space that neither intersects $S$ nor $F$, and consider the cone over $X$ consisting of all codimension 2 spaces spanned by $T$ and points in $X$. This cone is a hypersurface of degree $m$ in $\mathbb{P}^{g}$, and if we consider the cones over all curves of degree $m$ in $F$, including the singular and reducible ones, we get a linear system $\mathfrak{d}$ inside $\left|\mathscr{O}_{\mathbb{P}^{g}}(m)\right|$ (making a coordinate change and letting $x_{0}, \ldots, x_{g}$ be the coordinates of $\mathbb{P}^{g}$, we can assume that $F$ is given by $x_{3}=\cdots=x_{g}=0$ and $T$ given by $x_{0}=x_{1}=x_{2}=0$, in which case the cones are precisely the zero-sets of the homogeneous polynomials of degree $m$ in $x_{0}, x_{1}, x_{2}$, which is clearly a linear system). Since the base locus of $\mathfrak{d}$ is $T$ and $T \cap S=\emptyset$, then $\mathfrak{d}$ doesn't have base-points in $S$. We can hence apply Bertini's theorem and conclude that the general $\left.C \in \mathfrak{d}\right|_{S}$ is nonsingular. Each curve is connected because the number of connectedness components for each $C \in|m H|$ is constant and $\left.\mathfrak{d}\right|_{S} \subseteq|m H|$.

We now prove that $\operatorname{gon}(C)=(m-1) H^{2}=(m-1)(2 g-2)$. By Lemma 2.1, we see that $\operatorname{gon}(C) \leq(m-1) H . H=(m-1)(2 g-2)$. Now suppose that $|A|$ is a $g_{d}^{1}$ of minimal degree on $C$, and note that $|A|$ must be base-point free. Then $d \leq$ $(m-1)(2 g-2)$. Let $g^{\prime}$ be the genus of $C$. We have $2 g^{\prime}-2=(m H)^{2}=m^{2}(2 g-2)$, so that $g^{\prime}=m^{2}(g-1)+1$, and so we get $\rho(g, 1, d)=-m^{2}(g-1)+2 d-3$. Using that $d \leq(m-1)(2 g-2)$, we get $\rho(g, 1, d) \leq-(g-1) m^{2}+(4 g-4) m-4 g+1$, and by solving this as a second-degree equation in $m$, we see that this expression is always $<0$. (The expression $\rho(g, r, d)$ was defined in the introduction.)

Following the work of Lazarsfeld and Tyurin [10,13], for any base-point free line-bundle $A$ on $C \in|m H|$, there is defined a rank-2 vector bundle $\mathscr{E}_{C, A}$ by

$$
0 \rightarrow \mathscr{E}_{C, A} \rightarrow H^{0}(C, A) \otimes \mathscr{O}_{S} \rightarrow A \rightarrow 0,
$$


where $A$ is considered as a sheaf on $S$ by expansion by 0 . We have that $c_{1}\left(\mathscr{E}_{C, A}\right) \cong \mathscr{O}_{S}(C)$ and $c_{2}\left(\mathscr{E}_{C, A}\right)=d$. Furthermore, $\chi\left(\mathscr{E}_{C, A} \otimes \mathscr{E}_{C, A}\right)=$ $2-2 \rho(g, 1, d)$, and since $h^{2}\left(S, \mathscr{E}_{C, A} \otimes \mathscr{E}_{C, A}\right)=h^{0}\left(S, \mathscr{E}_{C, A} \otimes \mathscr{E}_{C, A}\right)$, it follows that $h^{0}\left(S, \mathscr{E}_{C, A} \otimes \mathscr{E}_{C, A}\right) \geq 1-\rho(g, 1, d)$. Since $\rho(g, 1, d)<0$ in our case, then we get that $\mathscr{E}_{C, A}$ is non-simple. By Green and Lazarsfeld [5, Lemma 3.1], and independently by Donagi and Morrison [3], any such non-simple vector bundle $\mathscr{E}_{C, A}$ lies inside an extension

$$
0 \rightarrow M \rightarrow \mathscr{E}_{C, A} \rightarrow N \otimes \mathscr{I}_{\xi} \rightarrow 0,
$$

where $M, N$ are two line bundles on $S$ satisfying $h^{0}(S, M) \geq 2, h^{0}(S, N) \geq 2$, $M \otimes N \cong \mathscr{O}_{S}(C),|N|$ is base-point free, and where $\xi$ is a 0-dimensional subscheme of finite length. We have $c_{2}\left(\mathscr{E}_{C, A}\right)=d=M \cdot N+\operatorname{length}(\xi)$.

Now, since $\operatorname{Pic}(S)$ is generated by $H$, we have $M \cong \mathscr{O}_{S}(a H)$ and $N \cong$ $\mathscr{O}_{S}(b H)$, where $a \geq 1$ and $b \geq 1$ since $h^{0}(S, M) \geq 2$ and $h^{0}(S, N) \geq 2$. It follows that $M \cdot N+\operatorname{length}(\xi)=a b H^{2}+\operatorname{length}(\xi) \geq a b(2 g-2)$. Since $M \otimes N \cong \mathscr{O}_{S}(m H)$, we have $a+b=m$, and so $a b \geq m-1$. We conclude that $d \geq(m-1)(2 g-2)$, as desired.

So gon $(C)=(m-1)(2 g-2)$ for all $C \in|m H|$. And so in particular, this applies when $C$ is the intersection of $S$ with a cone $Q$ over a general smooth curve $X$ of degree $m$ in the plane $F$. Now let $Z$ be one of the infinitely many codimension 2 subspaces of $\mathbb{P}^{g}$ that exist in $Q$, and let $\mathscr{H}_{Z}=\left|\mathscr{O}_{\mathbb{P}^{g}}(1) \otimes \mathscr{I}_{Z}\right|$, i.e., the linear system of all hyperplanes $J$ in $\mathbb{P}^{g}$ that contain $Z$. We show that the set

$$
\left|A_{Z}\right|=\left\{(J \cap C) \backslash(C \cap Z) \mid J \in \mathscr{H}_{Z}\right\}
$$

is a linear system of degree $(m-1)(2 g-2)$ on $C$.

It is clear that all the elements in $\left|A_{Z}\right|$ are linearly equivalent, since all elements in $\mathscr{H}_{Z}$ cut out linearly equivalent divisors on $C$. To find the degree of $\left|A_{Z}\right|$, it suffices to show that $\#(Z \cap C)=2 g-2$. To do this, consider a hyperplane $J \in \mathscr{H}_{Z}$. The points where this intersects $C$ are precisely the intersection of the varieties $J \cap Q$ and $J \cap S$. Now $J \cap Q=Z+V_{m-1}$ where $V_{m-1}$ is a hypersurface in $J$ of degree $m-1$. And $J \cap S=C_{2 g-2}$, a curve in $J$ of degree $2 g-2$. Since $Z$ and $C_{2 g-2}$ are both contained in $J$, then these intersect in $2 g-2$ points, as desired.

We now show that two distinct codimension 2 subspaces $Z$ and $Z^{\prime} \neq Z$ give divisors $A_{Z}$ and $A_{Z^{\prime}}$ that are not linearly equivalent. Suppose they were. Then $A_{Z}<\left.J_{1}\right|_{C}$ for some $J_{1} \in \mathscr{H}_{Z}$ and $A_{Z^{\prime}}<\left.J_{2}\right|_{C}$ for some $J_{2} \in \mathscr{H}_{Z^{\prime}}$. But since $\left.\left.J_{1}\right|_{C} \sim J_{2}\right|_{C}$ and we are assuming $A_{Z} \sim A_{Z^{\prime}}$, then $\left.J_{1}\right|_{C}-\left.A_{Z} \sim J_{2}\right|_{C}-A_{Z^{\prime}}$, and so $\left.\left.Z\right|_{C} \sim Z^{\prime}\right|_{C}$. But since $Z \neq Z^{\prime}$ and have $T$ as the only space of intersection, then $\left.Z\right|_{C} \neq\left. Z^{\prime}\right|_{C}$, and since we showed that each such codimension 2 subspace intersects $C$ in $2 g-2$ points and $m \geq 3$, then this contradicts the gonality of $C$.

It follows that each linear system $|A|, A \in\left\{A_{Z}\right\}_{Z}$, arises from a unique codimension 2 subspace $Z$ in $Q$, and so it follows that $\operatorname{dim} W_{\operatorname{gon}(C)}^{1}(C)=1$. In fact, we see from our construction that $W_{\operatorname{gon}(C)}^{1}(C)$ has an irreducible component isomorphic to the plane curve $X$. 
Note that the curves in this example are finite covers of plane curves.

Remark 2.2. In the case $S$ is a smooth quartic in $\mathbb{P}^{3}$ containing a line $\ell$, and $H$ is a plane section on $S$, then the curves $C \sim m H$ have gonality $<4(m-1)$ for $m \geq 5$. Indeed, by Proposition 2.1, putting $D_{1}=(m-1) H+\ell, D_{2}=H-\ell$ and using that $H^{2}=4$, we get $\operatorname{gon}(C) \leq D_{1} \cdot D_{2}=3 m$, which is $<4(m-1)$ whenever $m \geq 5$.

A more geometrical way to see this is that if $S$ contains a line $\ell$ and $C$ is a smooth curve in the linear system $|m H|$, then $\ell$ intersects $C$ in $m$ points, and so all planes going through $\ell$ define a $g_{d}^{1}$ on $C$ where $d=4 m-m=3 m$. (We actually have gon $(C)=3 \mathrm{~m}$. See Remark 2.4.).

We see that in these cases, the infinitely many codimension 2 subspaces $Z$ in the cone $Q$ of the proof of Theorem 1.1 no longer define pencils of minimal degree on $C$.

When $m \leq 4$ and $S$ is a smooth quartic in $\mathbb{P}^{3}$, we don't need any conditions on $\operatorname{Pic}(S)$, as we see in the next example.

Example 2.3. Let $S$ be any smooth quartic in $\mathbb{P}^{3}$, let $H$ be a plane in $\mathbb{P}^{3}$, and let $C$ be a smooth curve in $|m H|$, where $m \in\{3,4\}$. By Proposition 2.1, we see that $\operatorname{gon}(C) \leq(m-1) H . H=4(m-1)$. However, by [11, Theorem 4.12], since $C$ is a complete intersection, we have $\operatorname{gon}(C) \geq 4(m-1)$, and so equality follows.

We can now construct infinitely many $g_{4(m-1)}^{1}$ 's on $C$ precisely as we did in the proof of Theorem 1.1.

Remark 2.4. The lower bound for $\operatorname{gon}(C)$ found in [11] is as follows: If $C \in \mathbb{P}^{n}$ is a complete intersection of hypersurfaces of degree $2 \leq a_{1} \leq a_{2} \leq \cdots \leq a_{n-1}$ and $|A|$ is a $g_{d}^{1}$ on $C$, then $d \geq\left(a_{1}-1\right) \cdot a_{2} \cdots a_{n-1}$. So for $m \geq 5$ in Remark 2.2 , we get $d \geq 3 m$, so that the curves in those cases have gonality precisely equal to $3 \mathrm{~m}$.

Acknowledgements. The proof of this theorem is due to some interesting conversations with Gian Pietro Pirola for the case $H^{2}=4$, i.e., when $S$ is a quartic surface in $\mathbb{P}^{3}$. Thanks also to Andreas Leopold Knutsen for valuable comments.

Open Access. This article is distributed under the terms of the Creative Commons Attribution Noncommercial License which permits any noncommercial use, distribution, and reproduction in any medium, provided the original author(s) and source are credited.

\section{References}

[1] C. Ciliberto and G. Pareschi, Pencils of minimal degree on curves on a K3 surface, J. Reine. Angew. Math. 460, 14-36 (1995).

[2] M. Coppens and G. Martens, Secant spaces and Clifford's theorem, Comp. Math. 78, 193-212 (1991).

[3] R. Donagi and D. Morrison, Linear systems on K3 sections, J. Diff. Geom. 29, 49-64 (1989). 
[4] D. Eisenbud et Al., The Clifford dimension of a projective curve, Comp. Math. 72, 173-204 (1989).

[5] M. Green and R. Lazarsfeld, Special divisors on curves on a K3 surface, Inventiones Math. 89, 357-370 (1987).

[6] E. Kani, On Castelnuovo's equivalence defect, J. Reine Angew. Math. 352, 24-70 (1984).

[7] A. Knutsen, On kth-order embeddings of K3 surfaces and Enriques surfaces, Manuscripta Math. 104, 211-237 (2001).

[8] A. Knutsen, Gonality and Clifford index of curves on K3 surfaces, Arch. Math. 80, 235-238 (2003).

[9] A. Knutsen, On two conjectures for curves on K3 surfaces, Int. J. Math. 20, 1547-1560 (2009).

[10] R. Lazarsfeld, Brill-Noether-Petri without degenerations, J. Diff. Geom. 23, 299-307 (1986).

[11] R. LAZARSFeld, Lectures on linear series, with the assistance of G. F. del Busto, arXiv:alg-geom/9408011v1.

[12] B. Saint-Donat, Projective Models of K - 3 Surfaces, Amer. J. Math. 96, 602-639 (1974).

[13] A. Tyurin, Cycles, curves and vector bundles on an algebraic surface, Duke Math. J. 54, 1-26 (1987).

Nils Henry Williams Rasmussen

Department of Mathematics,

University of Bergen,

Johs. Bruns Gate 12,

5008 Bergen, Norway

e-mail: nilshwr@math.uib.no

Received: 9 November 2010 